Original Contribution

\title{
STUDY OF RESULTS BETWEEN INITIAL AND FINAL SPECIFIC TESTS IN WOMEN WITH NORMAL PREGNANCY
}

\author{
Ir. Nesheva* \\ Faculty of Public Health, Healthcare and Tourism, National Sports Academy "Vassil Levski", \\ Sofia, Bulgaria
}

\begin{abstract}
PURPOSE: The aim of this study was to achieve a control model of basic functional indicators established by comparative analysis between the results of initial and final anthropometric and functional tests in women with normal pregnancy.

METHODS: The research was done among 112 individuals, all Bulgarians, in the period 2015-2018, ages 21 to 31 years old. In order to fulfil the aim of the research we used: functional tests for assessing the effects of our original program build for the specific needs of pregnant women. The program content was branded under our family name: "Gym program for pregnant women Nesheva" and was validated in our doctoral thesis and then published in a book (under patent).

RESULTS: During our original gymnastics program was registered data by specific tests and the analysis has shown that the average values (Mean $\pm \mathrm{SD}$ ) of the measured parameters, in the three periods of time (during the pregnancy) are statistically different $(\mathrm{p}<0.05)$, which is a natural process resulting from the changes taking place in the body of the woman with the progression of pregnancy. These values are interpreted as optimal prenatal weight gain, but probably gymnastics for pregnant women also favour the maintenance of normal values. Studied indicators are presented with the results of the Mean \pm SD of the blood circulation parameters and the vital capacity of the pregnant women.

CONCLUSIONS: The results in our study are in support to establishing a system for collecting and processing information on the clinical status, general and functional characteristics of pregnant women is a reliable basis for validating standards for regulated inclusion in physical activity programs in normal pregnancy. Indicators of blood circulation and vital capacity in relative resting conditions of pregnant women when enrolled in the gymnastics program are within the limit of normal.
\end{abstract}

Key words: Pregnant women, gym exercises, functional status, anthropometric indicators, control model, arterial blood pressure, vital capacity

\section{INTRODUCTION}

Recreation activity, in particular, gym exercises during pregnancy, is an important component of the daily life of pregnant women in order to be born healthy and vigorous baby. The mother's wellness lifestyle will contribute she feel comfortable during pregnancy, delivery will take place more easily and help faster recovery in the period after birth (1).

\footnotetext{
*Correspondence to: Assoc. Prof. Irina Nesheva, Ph.D, National Sports Academy "Vassil Levski" Sofia, Faculty of public health, healthcare and tourism, Department Gymnastic Sports, Email: iranesheva2005@abv.bg
}

Those innovative tools to be healthy during the pregnancy are a modern recreation practice. The Recreation activities can be regarded as a tool for developing a polyvalent healthy, Wellness (Healthy life style) and SPA (Sanus per Aqua) culture (2). At the same time, it is important to discuss issues with remedial influence like the Recreation activity in an aquatic environment and the immediate connection with the improvement of the quality of life and health status for the participants (3). Staff competencies are the guaranty for the quality of offered recreation services. The quality services for pregnant women required staff with fellowships, 
training seminars or received certified competencies (4). Introducing Smart Apps in the certification process provides specialized training of personnel with specialized secondary and higher education (5). The intensity during the specific programs for pregnant women is very important to be well managed $(6,7)$. As well teachers should integrate an interactive approach in their classrooms and this will be a powerful tool for transforming any training into a dynamic learning environment (8, 9). After 2000, leading sports experts are distinguished from average models and point out that the process of building motor skills required to develop a system to control specialized motor skills, not only in sport but even in the physical culture or free time activities (10-12). In the Field of medical care for the pregnant women, most international experts are in absolute consensus on the positive effect of the recreation gym during the pregnancy (13).

\section{METHODOLOGY}

The research was done among 112 individuals (only 100 full test-retests): all Bulgarians, in the period 2015-2018, ages 21 to 31 years old. There were only pregnant women and we worked in partnership with the specialized hospitals "Sheinovo" and "Mother House (Maichin dom)". The average age of the researched individuals was 26 years. For the purposes of the study, the target group was divided into age groups (21 - 26 years; $27-31$ years). In the classes, the predominant number of registered pregnant women are in the second trimester (from IV to VI months), confirming their conscious awareness and claim to be the most suitable period for physical activity. Every pregnant woman gives us a confirmation recommendation of obstetrician-gynecologist and permission to attend our program. The research among the age groups was done in the biggest Sport University in Bulgaria (National sports academy "V. Levski", Sofia), and they sign a declaration for an agreement to give us permission for publishing the obtained results. In order to fulfil the aim of the research we used: functional tests for assessing the effects of our original program build for the specific needs of pregnant women. The program content was branded under our family name: "Gym program for pregnant women Nesheva" and was validated in our doctoral thesis and then published in a book (under patent).

A. Anthropometric indicators: height $(\mathrm{H})$; body weight (weight before pregnancy - W_I, weight for inclusion in the program - W_M, weight at the end of the program - W_F); body mass index before pregnancy (BMI); thoracic laps on initial (IT) and final examination (FT): respiratory pause - BPCC_I, Aspiration (inhalation) - ICC_I, exhalation - ECC_I; Aspiration (inhalation) - ICC_I, exhalation ECC_I; After aspiration (inhalation) pause BPCC_F, Aspiration (inhalation) - ICC_F, exhalation - ECC_F. The weight of each woman is determined in $\mathrm{kg}$ before pregnancy when enrolled in the program at the end of program participation before and after the work. A SEGA 761 medical weighing machine was used. Growth is individually measured in $\mathrm{cm}$ medical stadiometer SEGA 222. Through the method of centimetres bust (pause maximum inhalation, the maximum exhalation) were measured with centimetre unit $(\mathrm{cm}$.) of each woman individually upon entry into the program and final measurement ending participation in gymnastic activities.

B. Functional Characteristics: HR (HR HR), initial and final IT - FT (HR_I, HR_F), before and after a separate gymnastic exercise (HR pre, post), during exercise (HRmax, HRmin, HRavg); arterial blood pressure (systolic pressure - RRs, diastolic pressure - RRd) at baseline (RRs_I, RRd_I) and final study (RRs_F, RRd_F), before and after a separate gymnastic exercise (RRspre, RRdpre, RRspost, RRdpost); Experimental methods for the determination of morph-functional indicators, vital capacity (VC) at the start and stop testing (VC_I, VC_F), before and after separately gymnastic activity (VC pre, VC post); indirectly determined respiratory indicators (oxygen consumption - VO2peak, respiratory rate - R-Ravg, minute ventilation - VE peak) during a gymnastic exercise. Indicators circulatory heart rate and arterial blood pressure (systolic and diastolic) were measured in a sitting position with clinically certified equipment Omron M10-IT. Vital capacity was measured with the method of spirometry. An aqueous spirometer was used, the readings being read in $\mathrm{ml}$.

C. Statistics: The processing of the results was done with the Microsoft Office Excel 97-2003 program and the data were statistically processed with SPSS Statistics 19 by Descriptive Statistics, NPar Tests (Wilcoxon RT), Nonparametric Correlations (Spearman's rho), ANOVA with repeated measures. The verification of the normal distribution of data was through the Shapiro-Wilk and 
Kolmogorov-Smirnov test. Variation analysis (discriminative statistics) of the data obtained from the studies on tasks and directions. The critical rejection level of the zero hypothesis was set to 0.05 . Graphics are made with Microsoft Excel and Graph Pad.

The aim of this study was to achieve a control model of basic functional indicators established by comparative analysis between the results of the initial and final study of anthropometric and functional tests in women with normal pregnancy.

\section{RESULTS}

Mean values and standard deviations of age, height, body weight (baseline, weight at program start and ending) and body mass index before pregnancy for the women participating in the program are shown in Table 1.

Figure 1 presents the ANOVA with repeated measures of body mass index - pre-pregnancy weight, instantaneous weight at program startup and weight at program completion. The comparative analysis has shown that the average values (Mean \pm SD) of the measured parameters in the three periods of time (during the pregnancy) are statistically different ( $p$ $<0.05$ ), which is a natural process resulting from the changes taking place in the body of the woman with the progression of pregnancy.

Table 1. Values (Mean $\pm S D)$ of the anthropometric indicators age, height, initial, instantaneous and final weight, BMI.

\begin{tabular}{|c|c|c|}
\hline Indicators & $\mathbf{N}$ & Mean \pm SD \\
\hline (A) Age & 100 & $28,98 \pm 3,94$ \\
\hline (H) Height $[\mathrm{cm}]$ & 100 & $167,14 \pm 6,56$ \\
\hline (W_I) Body mass before pregnancy $[\mathrm{kg}]$ & 100 & $57,05 \pm 8,57$ \\
\hline (W_M) Body Mass on Program Inclusion [kg] & 100 & $62,33 \pm 9,28$ \\
\hline (W_TT) W eight at Program Termination, before Birth [kg] & 100 & $67,78 \pm 9,684$ \\
\hline (BMI) Pre-pregnancy Body Mass Index $[\mathrm{kg} / \mathrm{cm}]$ & 100 & $20.38 \pm 2.49$ \\
\hline
\end{tabular}

These values are interpreted as optimal prenatal weight gain probably gymnastics for pregnant women also favour the maintenance of normal values (14-18).

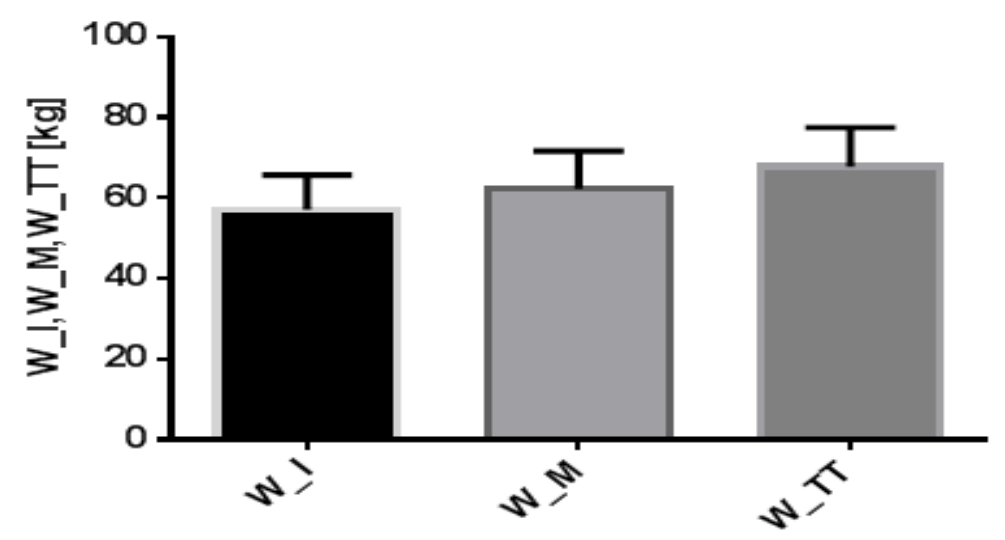

Figure 1. Mean values with ANOVA with repeated measures and SD of body mass index.

In Table 2 are presented the results of the Mean \pm SD of the blood circulation indicators and the vital capacity of the pregnant women during the gymnastics program.

The results obtained in relative resting conditions for HR, RRs and RRd show for: heart rate values in the upper limit of the norm, systolic pressure around the lower limit and mean for the normal diastolic pressure. The established status of blood circulation is consistent with published literature data (19). Vital capacity does not differ from that of healthy non-pregnant women (20). 
Table 2. Values (Mean $\pm S D$ ) of the functional parameters heart rate, arterial blood pressure (RRs, $R R d)$, vital capacity at entry into the program.

\begin{tabular}{lrc}
\multicolumn{1}{c}{ Indicator } & $\mathbf{N}$ & Mean \pm SD \\
HR $[\mathrm{bpm}]$ Heart rate & 100 & $87.9 \pm \mathbf{1 2 . 2 3}$ \\
RRs [mm Hg] Systolic blood pressure & 100 & $109.5 \pm 13.68$ \\
RRd [mm Hg] Diastolic blood pressure & 100 & $69.1 \pm 8.36$ \\
VC [m1] Vital capacity & 100 & $3038.5 \pm 525.55$
\end{tabular}

Table 3. Variance analysis and comparison of anthropometric and functional parameters between IT and FT.

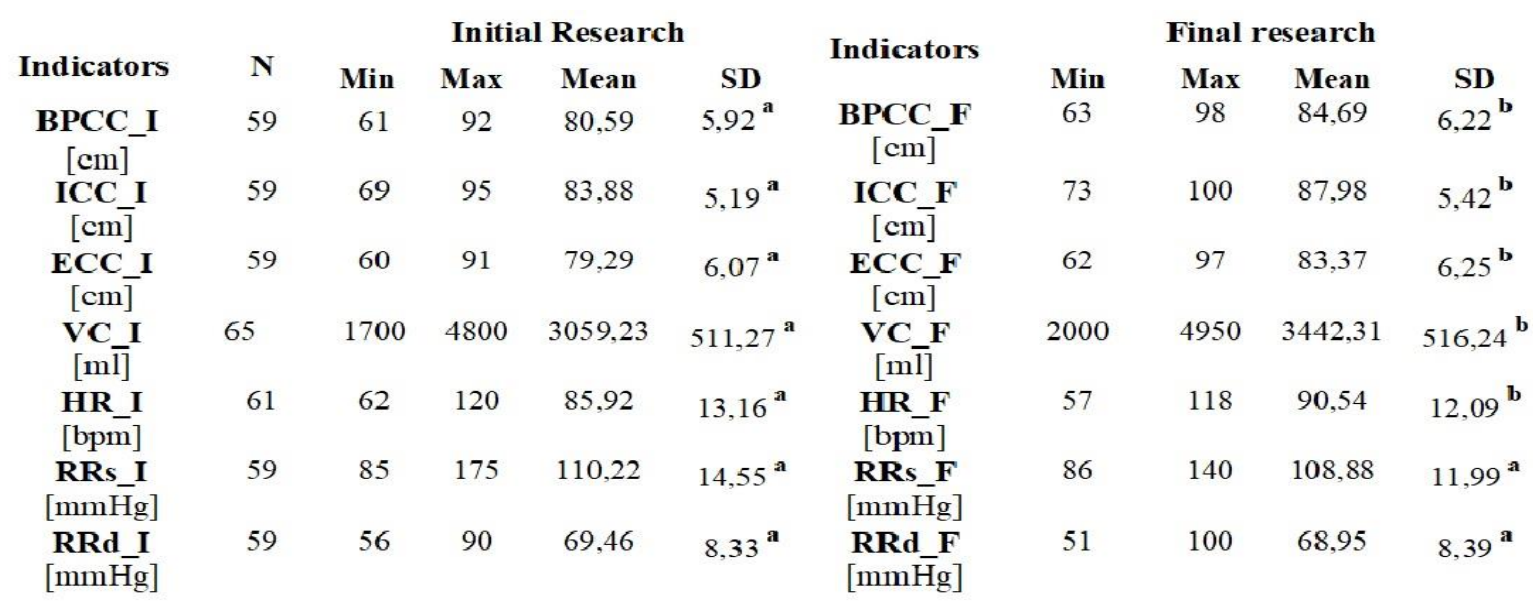

* Uniform letters mean lack of statistical differences, and the different - the presence of such; all significant differences found were at confidence level $\mathrm{p}<0.01$.

Table 3 presents data from the descriptive statistics (analysis of variance) and the comparative analysis between the initial and final testing of anthropometric and functional indicators thoracic laps (pause maximum aspiration (inhalation), maximal exhalation (Figure 2), vital capacity (Figure 3), cardiac frequency (Figure 4), and arterial blood pressure (systolic and diastolic).

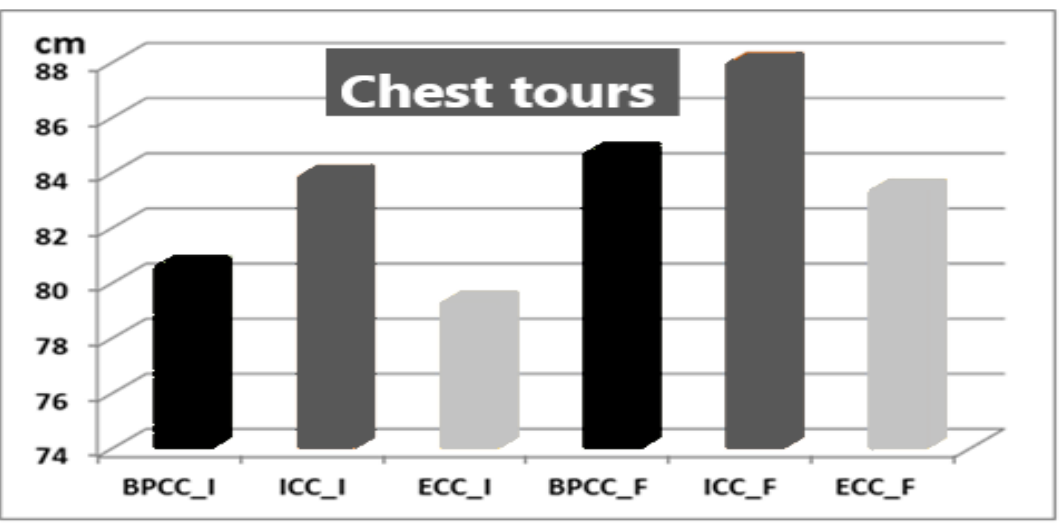

Figure 2. Comparison between IT and FT pause, aspiration, exhalation in pregnant women.

From the comparison identifies significant differences $(p<0.01)$ for the bust, vital capacity and heart rate with higher values in the final study (Figures 2, 3, 4). 


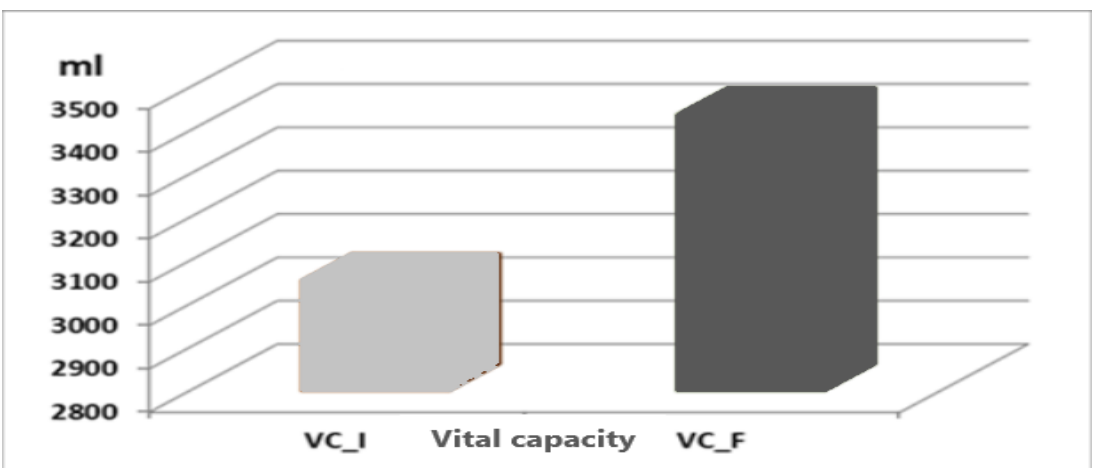

Figure 3. Comparison between IT and FT vital pause, aspiration, exhalation in pregnant women.

These respiratory values are an adequate response to the increased metabolic needs of women in the last trimester. The only exception is the comparison of the indicator arterial blood pressure (RRs and RRd), it does not establish a significant difference $(p>0.05)$.
This result confirms the thesis that the benefits of exercise improve blood pressure control and that gymnastics can reduce the risk of hypertension.

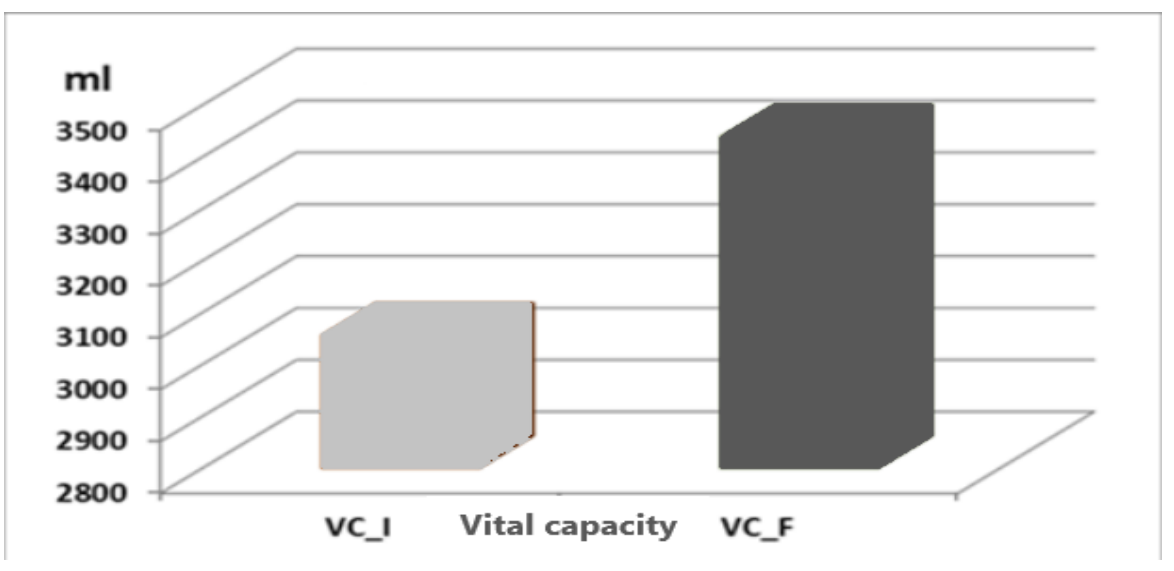

Figure 4. Comparison between IT at the initial and final testing.

In Figures 5 and 6 shows histograms that have a single symmetric pulse frequency distribution at IT (Mean - 87.9) and FT (Mean

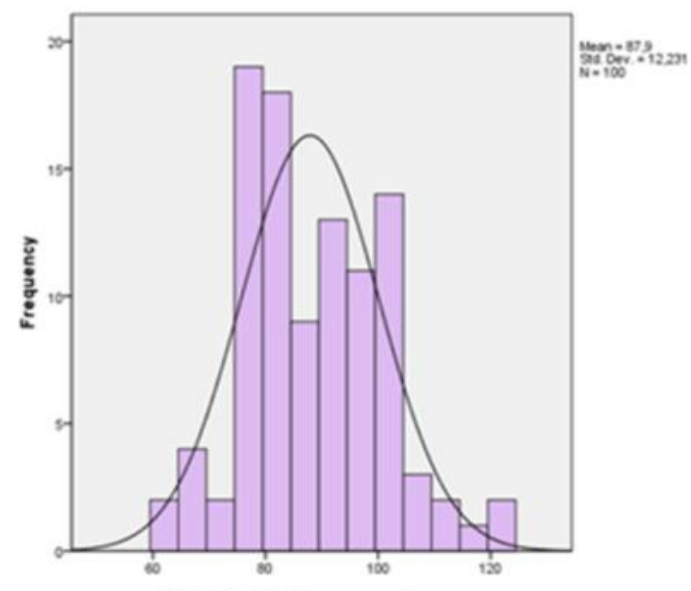

HR - Initial research

Figure 5 and

Cardiac Response Indicators RRs, RRd, HR, VC measured before (pre) and immediately
- 90.54). In summary, the data give reason to believe that pregnant women maintain their functional reserve.

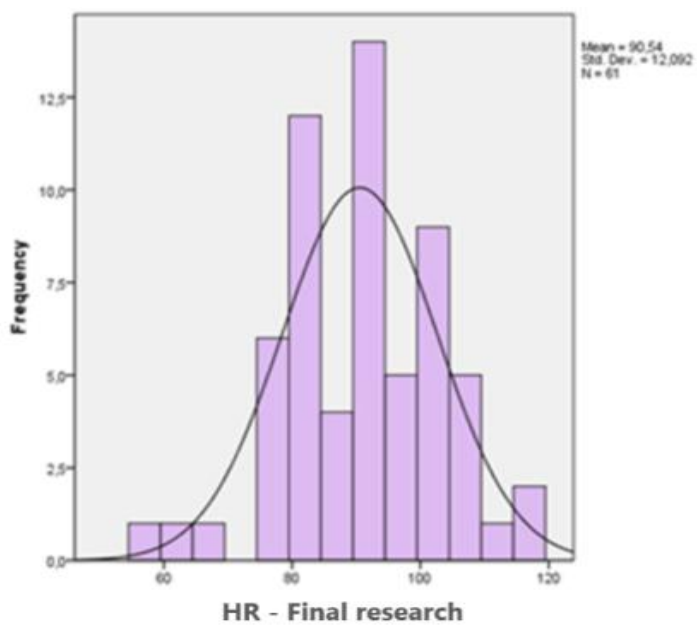

Figure 6. "Gaussian curves" of HR and FT Heart Rate.

after (post) specific gymnastic occupation, BPCC, ICC and ECC are presented in Table 4. 
Table 4. Variation of Mean $\pm S D$ of Cardio Respiratory Indicators.

$\begin{array}{lcc}\quad \text { Indicators } & \text { Mean } & \text { SD } \\ \text { RRs pre [mm Hg] - Systolic blood pressure before the program } & 101,67 & 9,31 \\ \text { RRs post [mm Hg] - Systolic blood pressure after the program } & 98,17 & 7,49 \\ \text { RRd pre [mm Hg] - Diastolic blood pressure before the program } & 61,00 & 4,69 \\ \text { RRd post [mm Hg] - Diastolic blood pressure after the program } & 59,33 & 5,12 \\ \text { HR pre [bpm] - Heart rate before the program } & 79,50 & 10,46 \\ \text { HR post [bpm] - Heart rate after the program } & 82,17 & 8,35 \\ \text { VC pre [ml] - Vital capacity before the program } & 3000 & 457,16 \\ \text { VC post [ml] - Vital capacity after the program } & 3117 & 465,48 \\ \text { BPCC [cm] - chest circumference during breathing pause } & 78,50 & 1,76 \\ \text { ICC [cm] - chest aspiration (inhalation) } & 82,25 & 2,66 \\ \text { ECC [cm] - chest during exhalation } & 76,75 & 2,09\end{array}$

Significant differences were found between body mass measured at different times and corresponding BMI $(\mathrm{p}<0.05)$. The comparison between the indicators measured before and after the gymnastic exercises showed that only the values of diastolic pressure and vital capacity $(\mathrm{p}<0.05)$ were significantly different.

\section{DISCUSSION}

The correlation data analysis reveals the following most significant relationships at the significance level (0.05 and 0.01) - in our study we find positive correlations between the indicators weight, height and BMI. The indicator chest trips (breasts) correlate with each other and with BMI positively. The influences of gymnastic exercises on indicators of blood circulation are sustainable and healthy. The results of the study at a gymnastic activity are presented as an example of changes in heart rate and blood pressure analysed complexed with test respiratory and sports-pedagogical indicators (the data will be presented in other publications). The systolic pressure and heart rate after the gymnastic exercise correlate with the systolic pressure and heart rate measured after the exercise; prediastolic pressure correlates only with diastolic pressure after gymnastics; the vital capacities correlate positively, the pre-excavated heart rate has no significant correlations, the BMI determined on the day of the study shows significant positive correlations with $\mathrm{VC}$ pre and $\mathrm{VC}$ post. The results cannot be compared with data from other authors because they are specifically adapted for this contingent. Systolic pressure is a little low compared to reference values for women, which is probably related to the state of pregnancy. Diastolic pressure and heart rate are within the limit of normal. Vital capacity is not significantly different from that of untrained women. The three values of BMI in the normal range for women, although pregnancy. An interesting result is the increased vital capacity after gymnastics. The stimulatory effect of gymnastic exercises on respiratory function is essential for pregnant women. The applied model for women with normal pregnancy activates appropriate cardiorespiratory functions that are restored rapidly to baseline. The results obtained suggest that the gymnastic load from the innovative model has a beneficial effect on cardiorespiratory function and maintains the physical fitness of women with normal pregnancy.

\section{CONCLUSIONS}

The results obtained for the influence of the applied gymnastics program Nesheva for prophylactic direction and functional control in normal pregnancy, analyzed in accordance with the established unexplained and scarcely illuminated problems in literary sources, justifies the following main conclusions:

1. The established system for collecting and processing information on the clinical status, general and psychometric characteristics of pregnant women is a reliable basis for validating standards for regulated inclusion in physical activity programs in normal pregnancy.

2. Indicators of blood circulation and vital capacity in relative resting conditions of pregnant women when enrolled in the gymnastics program are within the limit of normal.

3. Upon completion of the Nesheva program, a significant increase in chest circumferences, vital capacity, heart rate, and improved blood pressure control has been identified.

4. Upon completion of the Nesheva program, a significant increase in chest circumferences, vital capacity, heart rate, and improved blood pressure control has been identified. 
Table 5. Abbreviations table content

$\begin{array}{ll}\text { Abbreviations } & \text { Full description of the indicators } \\ \text { (RR) } & \text { Aiterial blood pressure } \\ \text { (RRd I) (RRd_F) } & \text { Diastolic blood pressure - IT, FT } \\ \text { (RRdpost) } & \text { Diastolic blood pressure after exercise } \\ \text { (RRdpre) } & \text { Diastolic blood pressure before exercise } \\ \text { (RRs_I)(RRs_F) } & \text { Systolic blood pressure - IT, FT } \\ \text { (RRspost) } & \text { Systolic blood pressure after exercise } \\ \text { (RRspre) } & \text { Systolic blood pressure before exercise } \\ \text { (R-Ravg) } & \text { Respiratory Frequency } \\ & \text { Respiratory Frequency average } \\ \text { (VC) } & \text { Vital capacity } \\ \text { (VC post) } & \text { Vital capacity after occupation } \\ \text { (VC pre) } & \text { Vital capacity before occupation } \\ \text { (VC_I)(VC_F) } & \text { Vital Capacity - IT, FT } \\ \text { (VE) } & \text { Pulmonary ventilation } \\ \text { (VO2)(VO2 peak) } & \text { Oxygen consumption } \\ \text { (W_I) } & \text { Bodyweight before pregnancy } \\ \text { (W_M) } & \text { Body Mass on Entry into the Program } \\ \text { (W_TT) } & \text { Weight at Program Termination, before Birth } \\ & \end{array}$

\section{REFERENCES}

1. Nesheva, I., P. Somlev, E. Pavlova, Uzunova, G. Prilozhenie na ustroisstvoto Suunto Memory Belt po vreme na gimnastika za bremenni zheni. Sport \& Nauka [Application of the Sunto Memory Belt during gymnastics for pregnant women]. Sports \& Science Journal, 4: 7279, 2013. (In Bulgarian)

2. Dimitrova, B. New smart educational model - Wellness instructor. Avangard Prima, Sofia, Bulgaria, 2019.

3. Dimitrova, B. et al. Wellness TrainerIn Kompetenzstandard: von Lernenden zu erwerbende einheitliche ergebnisse, Kenntnisse intellektuellen \& praktischen Fähigkeiten. [Wellness Instructor Competence standard: uniform results to be acquired by students, knowledge of intellectual and practical skills]. Avangard Prima, Sofia, Bulgaria, 2018. (In German)

4. Dimitrova, B. Doklad - samootsenka za programna akreditatsiya $\mathrm{v}$ profesionalno napravlenie 7.5. Zdravni grizhi, spetsialnost Spa kultura, hidro, lito i talaso praktiki [Self-assessment report for program accreditation in a professional field 7.5. Health Care, specialty Spa Culture, Hydro, Litho and Thalasso Practices]. Avangard Prima, Sofia, Bulgaria, 2014. (In Bulgarian)

5. Dimitrova, B. Magistŭrska programa po SPA kultura, hidro, lito i talaso praktiki, s prodŭlzhitelnost 2 godini [Master's Program in SPA culture, hydro, lytho and thalasso practices, with two years duration]. Avangard Prima, Sofia, Bulgaria, 2014. (In Bulgarian)
6. Dimitrova, B. Synchronized swimming, a theoretical concept of the critical training zone. Sport Mont, XI(37-38-39), 41-47, 2013.

7. Dimitrova, B. Opit za vŭvezhdane na ponyatieto kritichna skorost $\mathrm{v}$ upravlenieto na natovarvaneto. Sport $i$ nauka [An attempt to introduce the concept of critical speed in training management], Sport and Science, XLXII, 5: 97-102, 2001. (In Bulgarian)

8. Dimitrova, B. Intelektualni i prakticheski umeniya za formiranite otnosheniya $v$ yadro vodni sportove. Sport i nauka [Intellectual and practical skills for formed relationships in unit water sports], Sport and Science, 4: 42-47, 2010. (In Bulgarian)

9. Dimitrova, B. et al. Multilingual app content for Android and iPhone presenting the "wellness instructor" VET course standard. Avangrd Prima, Sofia, Bulgaria, 2018.

10.Tatar, N. (2013). Physical activity in the free time of young people inMontenegroo. Sport Mont, XI(37-38-39), 241-246.

11.Trendafilov, D., Dimitrova, B. Aqua Spinning as anti-stress health prevention. Sport Mont, XI (37-38-39): 467-473, 2013.

12.Valev, Y. Kontrol i otsenka na sportnata podgotovka $\mathrm{v}$ konniya sport [Control and evaluation of sports training in equestrian sport]. NSA "V. Levski", Sofia, Bulgaria, 2015. (In Bulgarian)

13. American College of Obstetricians and Gynecologists. Exercise during pregnancy andthe postpartumm period. Obstet Gynecol, 99, 171-3, 2002.

14.Freisling, H., Elmadfa, I. \& Gall, I. 2006. The effect of socioeconomic status on dietary intake, physical activity and Body Mass Index in Austrian pregnant women. $J$ Hum Nutr Diet, 19, 437-445.

15.Jain, D., Khuteta, R., Chaturvedi, V., Khuteta, S. Effect of body mass index on pregnancy outcomes in nulliparous women delivering singleton babies:an observational study. Journal Obstet Gynecoll India, 62, 429-431, 2012.

16.Melzer, K., Schutz, Y., Boulvain, M. \& Kayser, B. Physical activity and pregnancy: cardiovascular adaptations, recommendations and pregnancy outcomes. Sports Med, 40: 493-507, 2010a.

17.Melzer, K., Schutz, Y., Soehnchen, N., Othenin Girard, V., Martinez De Tejada, B., Pichard, C Irion, O., Boulvain, M. \& Kayser, B. Prepregnancy body mass index 
and resting metabolic rate during pregnancy. Ann Nutr Metab, 57: 221-227, $2010 b$.

18.Montpetit, A. E., Plourde, H., Cohen, T. R. \& Koski, K. G. Modeling the impact of prepregnancy BMI, physical activity, and energy intake on gestational weight gain, infant birth weight, and postpartum weight retention. Journal Phys Act Health, 9: 1020-1029, 2012.
19.Gavriı̌ski V., D.Stefanova, E. Kiselkova \& Bichev, K. Fiziologiya na choveka s fiziologiya na sporta, chast II, Novi znaniya [Physiology of the man with the physiology of sport, part II], New knowledge, Sofia, Bulgaria, 2005. (In Bulgarian)

20.Wolfe, L. A., Weissgerber, T. L. Clinical physiology of exercise in pregnancy: a literature review. Obste Gynecolol Journal Canada, 25: 473-83, 2003. 
NESHEVA IR.

Trakia Journal of Sciences, Vol. 17, № 2, 2019 